\title{
Based on the multi-level optimization model of remanufacturing and pricing balance strategy research under TIFR subsidy in China
}

\author{
Shengyi Wang ${ }^{1, a *}$, Yan $\mathrm{Li}^{2}, \mathrm{~b}, \mathrm{Xin} \mathrm{Jin}^{3, \mathrm{c}}$ \\ ${ }^{1}$ Reading Academy, Nanjing University of Information Science \& Technology, Nanjing, China \\ ${ }^{2}$ School of Management Science and Engineering \& China Institute of Manufacturing Development, Nanjing University of \\ Information Science \& Technology, Nanjing, China \\ ${ }^{3}$ Eberly College of science, Pennsylvania State University, State College, America
}

\begin{abstract}
Remanufacturing is one of the most effective forms of the recycling economy, and the "Trade-in for Remanufacturing" (TIFR) program in China plays an important role in the development of remanufacturing. To understand this new business model of remanufacturing, the price and demand relationship of the NP and RP are examined in two periods, considering TIFR subsidy which is in practice in China. Comparing integrated optimization scheme and tiered optimization scheme, this paper then researches the pricing strategy with TIFR subsidy respectively, as multi-object optimization is employed in the latter scheme. Tiered optimization pricing is found to improve the old customer's TIFR enthusiasm, balance the remanufacturing system and work better with TIFR subsidy through numerical experiments. This paper studies the impacts of subsidy rate, receptiveness to RP, and the mixture of them, then verifies the proposed model and gives an inspiration to the management of the new remanufacturing business model about TIFR subsidy.
\end{abstract}

\section{INTRODUCTION}

As a significant part of product life cycle (PLC), remanufacturing is the management system which was firstly proposed in China in 1999 to meet demands of finance and environment. Different from the traditional manufacturing industry that focuses on production and distribution of new products (NPs), remanufacturing starts from the collection of used products, then disassembling, cleaning, inspecting, classifying remanufacturing parts, selecting remanufacturing technology, remanufacturing, and inspecting. The National Development and Reform Commission (NDRC) of Chinese government encouraged automobile manufacturers to build the complete recycling system for remanufacturing of used motor vehicle parts, and asked the remanufactured products (RPs) to have the same quality characteristics as the new products [1]. Besides that, the NDRC also declared that Chinese auto parts remanufacturing industry which is in an unprecedented development period [2].

Remanufacturing industry is the new development direction to promote circular economy and satisfy energy conservation and emission reduction. Compared with new products, remanufacturing products can save $50 \%$ of the cost, $60 \%$ of the energy, and $70 \%$ of materials. The remanufacturing products of machine tool, engineering machinery and auto parts have the greatest development potential in the market [3]. As the country with highest production capacity in the world, Chinese government has been setting up the remanufacturing policy system to regulate the remanufacturing industry in the market[4]. Although there exist some problems of environment which were caused by development of remanufacturing, many researchers are exploring solution to change the status quo.

The price of RPs is obviously affected by the quality [5]. In addition, the demand of market, environment, and the government subsidy are also significant elements to change the price of remanufactured items. For environment factors, resources shortage and environmental pollution are still the most serious problems in the society. Although many experts and researchers have found methods to solve, the situation of environment is not positive which to some extent reflect the necessary of remanufacturing [6]. Therefore, the whole process of remanufacturing needs to be perfected, and improves the technology to reduce consumptions and emissions. Expanding the PCL is an important part in protecting the environment. However, for government subsidy, the capacity of remanufacturing industry and the prices of remanufacturing products will decrease with the increase of government subsidy. Besides that, the number of NPs may increase as the subsidy rises to meet the demand of collection of used products (UPs) [7]. The education level of consumers is a significant topic which is discussed recently. People with higher education prefer to buy RPs which may result in manufactures having 
to pay more expense to suppliers[8]. Thus, the awareness of people's environment protection will increase.

Trade-in for Remanufacturing (TIFR) is a much useful method to collect UPs to carry out remanufacturing[5]. Manufacturers or third-party recycling channels recycle UPs from consumers and give them equivalent items to stimulate their motivation, which is a common way to trade in the old for the new. Government TIFR subsidies and policies promote the development of remanufacturing industry, but different policies will bring different benefits to remanufacturer[7]. Therefore, optimal recovery decision is still a research object to be studied in different situations. Different from traditional method of TIFR, e-commerce platforms create a new way to recycle UPs by giving electronic coupon and advance payment, which can reduce the offline workload of remanufacturers, Such as Alibaba Group, JD and SUNING. Thus, TIFR is needed to figure out an optimal strategy to meet the profits of manufacturers and consumers. This essay studies the optimal decisions for the manufacturers and address the following managerial concerns:

1) What are the impacts of tiered pricing systems under integrated optimization scheme and tiered optimization scheme? Which one is better in pursuit of short-time profits or RP supply-demand balance?

2) What are the impacts of the TIFR subsidy on the different pricing decisions and the demand of the NP and RP?

3) How do customer's receptiveness to RP affect firm's decisions? What is the joint effect of customer's receptiveness to RP and subsidy on firm performance?

4) How enterprises can make use of the proposed model to adjust remanufacturing strategy

The organization of the paper is as follows. In Section 2, we review the literature. Section 3 introduces the analysis framework and discusses the price-demand relationship in the segmented market. Section 4 presents the profit-maximization model with TIFR subsidy under Integrated Optimization Scheme and Tiered Optimization Scheme, and identifies the multi-object optimal solutions. To validate our model and derive managerial implications, we conduct numerical studies in Section 5. Finally, we present concluding remarks and future research directions in Section 6.

\section{RELATED LITERATURE}

In this section, we review the literature related to two influencing factors of remanufacturing: The subject of first is pricing strategy which is the main research objectives in this essay. The reminder of section is the government legislation in China.

\subsection{Pricing Decisions of Remunufacturing}

There are different kinds of pricing decisions of remanufacturing, i.e., UP acquisition pricing, RP sales pricing, and trade-in replacement pricing.

UP acquisition pricing focused on market-driven recovery system and incentive mechanisms. Xiong et al proposed a buy-back acquisition pricing model with uncertain quality [9]. Wei et al derive collection incentive pricing strategies under the assumption of quality following a uniform distribution and partitioned [10]. Li et al compare the original equipment manufacturer (OEM) and the third-party remanufacturer (3PR) in different UP acquisition pricing models between economy and environment [11]. Liu et al. study optimal recovery of UP pricing decision based on convex collection [12].

Most RP sales pricing put emphasis on competitive RP and NP environment. Abbey \& Blackburn [34] investigate the optimal pricing of NP and RP using a model with the assumption that the customer does or does not show preferences for NP. Bittar explores RP pricing strategy by investigating factors that are important to leverage RP sales, brand equity and consumers' environmental consciousness [13]. Liu et al combine RP price with the variation of warranty period to get the optimal strategy [14]. Kleber et al study pricing strategy with the assumption that a consumer's willingness-to-pay (WTP) for a RP is a variable fraction instead of a fixed fraction of his/her WTP for the corresponding NP [15]. Gan et al discuss the impact of single channel and direct channel on the sales price of RPs, and the direct channel is where manufacturers sell RPs directly to consumers [16].

There are still some trade-in replacement pricing decision studies. Han et al derive the TIFR strategy considering consumer's RP acceptance and NP durability [17]. Ma et al consider pricing decision of TIFR with quality effect and price effect [5]. Xiao et al compare the propensity of manufacturers and retailers to TIFR for traditional retail channel and dual channel [18].

But most of the pricing decisions' objective is to maximize the profits, the balancing problems are seldom concerned.

\subsection{Government policies in China}

A Yuan et al analyze the system of government polices of remanufacture in China, and compare it with foreign countries to get possible future direction of Chinese remanufacturing [4]. Wang et al deploy systemic dynamics to examine the impact of different government subsidies, including initial subsidy, recycle subsidy, research and development subsidy and production subsidy on remanufacturing [19]. An investigation has been conducted by Zhang et al to understand the development status of the Chinese automotive parts remanufacturing industry, and they conclude that the government needs to improve policies, market supervision, and policy incentives [20]. The government should define attitudes towards the market competition and supervise the changes of market competitiveness based on market competition and consumer acceptance of RPs [21]. Cao et al. describe the development of the Chinese government's remanufacturing policy and divide it into three stages. They enumerate some problems of remanufacture and offer corresponding solutions to government [22]. Qiao \& Su study the impact of government subsidies on the remanufacturing activities of two kind manufacturers: OEM and independent remanufacturer (IR) [7]. However, the government 
subsidy is not the best way to increase economy and decrease environmental pollution.

The economy and environment are two significant objects of government's remanufacturing policy. Besides that, it is the most common way to stimulate the development of remanufacturing by subsidy and fund policies. Government fund policies are often better for retailers to remanufacturing. Right fund policy parameters tend to bring more benefits for all parts of closed-loop supply chain [23]. However, government still need to improve the resource efficiency of remanufacturing which has responsibility to promote remanufacturing production by policies and legislations [24]. In addition, social donation is a useful method for government to set subsidy policies to encourage which plays an important role to improve the economic performance [25]. Government fund policy encourages manufacturers to recycle and remanufacture waste electrical and electronic equipment (WEEE), but high subsidy policy often has a negative effect on the environment [26]. Carbon emission is a main environmental problem of remanufacturing. The policy of carbon regulation by government can effectively promote remanufacturing, though it will affect remanufacturers' profits [27]. Miao et al analyze the government subsidy of TIFR can reduce the loss of profits under carbon regulation. Except for carbon regulation, carbon tax and take-back legislation are also two significant way for government to manage corporations and reduce environmental pollution. Carbon emission reduction (CER) can stimulate remanufacturing in the competitive environment [28].

As consumption subsidies, the TIFN and TIFR subsidy are widely pushed through. Shu et al investigate two subsidy mechanisms: tax rebate and fixed unit trade-in subsidy [29]. Wang and Chen categorize the government legislations related to remanufacturing in China, and note that trade-in can promote recycle of UPs [30]. Ma et al focus on the influences of "trade-in for new" consumptionsubsidy in a closed-loop supply chain, compare the two cases of consumers with or without government subsidy, and find that all consumers are beneficiaries of the consumption-subsidy [31]. Han et al study TIFR subsidy by considering other influencing factors, but this subsidy is unit fixed and not proportional to the replacement price as applied in China [17].

Therefore, the TIFR subsidy applied in China based on the replacement price is still not involved by now, and the remanufacturing and pricing policy to balance the supply and demand of RP are further need to be strengthened.

\section{Model Description And Notations}

We examine products with limited lifetime and develop a two-period model links the original manufacturing period and the manufacturing/remanufacturing period. The manufacturer produces and markets only new products in period 1. As the quality or functionality of the products deteriorate with time, if the manufacturer offered TIFN and TIFR programs in period 2, old consumers have the options to trade in their UPs for the NP or RP before it reaches endof-life, while new consumers can only buy NP or RP.
In Section 3.1, we examine tiered quality collection \& remanufacturing system and market structure with TIFN and TIFR programs. And model notations are listed in Section 3.2. Then, we derive the demand functions in Section 3.3 for the NPs and RPs so as to develop optimal operational strategies in Section 4.

\subsection{Tiered Quality Remanufacturing System and Market Structure}

The potential market in period 1 is the original Market $O$ with no UP returned or replaced. The manufacturer only produces and markets new product in this period. Due to different degrees of wear and tear, the quality of UPs in period 2 varies significantly. We classify them into $n$ quality levels and denote them as $L_{i}(i=1,2, \ldots, n)$ with $L_{1}$ being the highest quality level and $L_{n}$ the lowest. Depending on the level of quality, different proportion $\left(\omega_{i}\right)$ of collected UPs $\left(L_{i}\right)$ can be remanufactured into RPs. Some old customers with $L_{i}$ products may come back to trade in for a NP or RP in Period 2, so there are $n$-level RP market $\alpha_{i}, i=1,2, \ldots, n$. The customers who didn't buy in Period 1 could purchase NP in Period 2 and form Market $\beta$. The market segmentation is shown in Fig. 1. The manufacturer offers unlimited NP but limited total number of RPs to each market. The old customers who choose to trade in for RP are offered a TIFR price and will get TIFR subsidy $(\theta)$ from the government, and those who choose for TIFN will only obtain an rebate by returning UPs. Fig.1 shows the relationship between the production and remanufacturing processes.

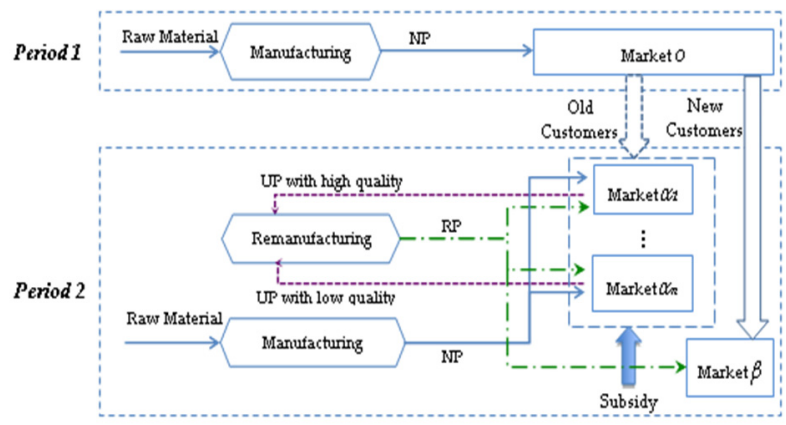

Figure 1. The tiered collection and remanufacturing system with TIFR subsidy

\subsection{Model Notations}

To facilitate an understanding of the model, we provide the symbols used and their corresponding meanings as follows:

$M$ :The potential market size in Period 1 (i.e. Market O).

$D_{O N}$ : Total size of customers who purchased NP in Period 1, which is total size of old customers in Period 2;

$L_{i}$ :The quality level of old customer's UP in Period 2 $(i=1,2, \ldots, n)$ with $L_{1}$ as the highest quality;

$\lambda_{i}$ :Proportion of old customers belonging to Market $\alpha_{i}$ in Period 2, i.e., the size of Market $\alpha_{i}$ is $\lambda_{i} D_{O N}\left(\sum_{i=1}^{n} \lambda_{i} \leq\right.$ $1)$;

$D_{\alpha N i}$ :Demand for NP from old customers in Market $\alpha_{i}$ in Period 2; 
$D_{\alpha R i}$ :Demand for RP from old customers in Market $\alpha_{i}$ in Period 2;

$D_{\beta N}$ : Demand for NP from new customers in Market $\beta$ in Period 2;

$D_{\beta R}$ : Demand for RP from new customers in Market $\beta$ in Period 2;

$v_{N}$ : Customer's willingness to pay (WTP) for NP in Period $1 \& 2\left(v_{N} \sim \mathrm{U}[0,1]\right)$;

$\delta$ : Ratio of consumers' WTP between RP and NP (customers' receptiveness to reman), i.e., the customer's WTP for a RP in Period 2 is $\delta v_{N}$ with his or her WTP for a NP is $v_{N}$;

$\theta: \quad$ TIFR consumption subsidy rate;

$c_{N}$ : Unit cost of manufacturing a NP in both periods;

$c_{\alpha i}$ : Remanufacturing cost of a UP with quality level $L_{i}\left(c_{\alpha 1} \leq c_{\alpha 2} \leq \ldots \leq c_{\alpha n} \leq c_{N}\right)$;

$\omega_{i}$ : Proportion of UPs with quality level $L_{i}$ that can be remanufactured in Period $2\left(\omega_{1} \geq \omega_{2} \geq \ldots \geq \omega_{n}\right)$;

$p_{N}$ : Unit price of a NP in period $1 \& 2$, decision variable;

$P_{\alpha R i}$ : Unit replacement price of RP that consumers need to pay after returning UP with quality level $L_{i}$ in Period 2, decision variable;

$P_{\beta R}$ : Unit price of a RP that consumers need to pay without returning UP in Period 2, decision variable, decision variable;

$r_{\alpha i}$ : Rebate that consumers will obtain when returning a UP with quality level $L_{i}$ in Period $2\left(r_{\alpha 1} \geq r_{\alpha 2} \geq \ldots \geq\right.$ $\left.r_{\alpha n}\right)$

$\pi$ : Total profit of manufacturer in both periods.

The superscripts $I, I(k), I^{\prime}$, denote the variables in the models under integrated optimization scheme, the superscripts $T, T(k), T$, denote those under tiered optimization scheme, and the superscripts * denotes optimal strategies and the results from corresponding models.

\subsection{Demand Analysis}

In Period 1, only NPs are available. The consumer's surplus from purchasing a NP in period 1 is $u_{O N}=v_{N}-$ $p_{N}$. Since a customer's WTP for NP $v_{N}$ is uniformly distributed on $[0,1]$ in Market $O$, the demand of the NP in Period 1 is

$$
D_{O N}=\left(1-p_{N}\right) M
$$

To estimate customer's demand for NP and RP in Period 2, we compare the consumer's surpluses of these two products in Market $\alpha_{i} \& \beta$. Between trading in and purchasing, customers will choose the one with the higher surplus. Since the out-of-pocket costs are $p_{N}-r_{\alpha i}$ of getting a NP and $(1-\theta) p_{\alpha R i}$ for old customers in Market $\alpha_{i}$, therefore old customer's surplus of trading in is $u_{\alpha N i}=$ $v_{N}-\left(p_{N}-r_{\alpha i}\right)$ for NP and $u_{\alpha R i}=\delta v_{N}-(1-\theta) p_{\alpha R i}$ for RP in Market $\alpha_{i}$ if TIFR subsidy rate $\theta$ is offered in period 2; while the consumer surplus is $u_{\beta N}=v_{N}-p_{N}$ for NP and $u_{\beta R}=\delta v_{N}-p_{\beta R}$ for RP in Market $\beta$. Be aware that the TIFR subsidy is a percentage of discounts applied to TIFR customers, hence it only increase the surplus of corresponding choice.
We assume the customer's WTP keep unchanged in both periods. Thus for old customers in Market $\alpha_{i}$, their WTP for NP is uniformly distributed on $\left[p_{N}, 1\right]$ in Period 2. Specifically, when $v_{N}=v_{\alpha i}^{\prime}=\frac{\left(p_{N}-r_{\alpha i}\right)-(1-\theta) p_{\alpha R i}}{1-\delta}$, the old consumer is indifferent between trading in for a NP and a $\mathrm{RP}$ in period 2 as both have equal surplus. When $v_{N}=$ $v_{\alpha i}^{\prime \prime}=\frac{(1-\theta) p_{\alpha R i}}{\delta}$, the consumer is indifferent between TIFR and keeping the UP. To make sure there must be some part of the customers to take TIFN or TIFR in Market $\alpha_{i}$, the pricing strategy $\left(p_{N}, p_{\alpha R i}\right)$ need to satisfy $v_{\alpha i}^{\prime}>p_{N}$, which implies $v_{\alpha i}^{\prime \prime}<p_{N}$. Since all the customers' WTP in Market $\alpha_{i}$ are no less than $p_{N}$, the demands for NP and RP in Market $\alpha_{i}$ are

$$
\begin{gathered}
D_{\alpha N i}=\lambda_{i} D_{O N} \frac{1-v_{\alpha i}^{\prime}}{1-p_{N}}=\lambda_{i} M[1- \\
\left.\frac{\left(p_{N}-r_{\alpha i}\right)-(1-\theta) p_{\alpha R i}}{1-\delta}\right](i=1,2, \cdots, n) \\
D_{\alpha R i}=\lambda_{i} D_{O N} \frac{v_{\alpha i}^{\prime}-p_{N}}{1-p_{N}}= \\
\lambda_{i} M\left[\frac{\left(p_{N}-r_{\alpha i}\right)-(1-\theta) p_{\alpha R i}}{1-\delta}-p_{N}\right](i=1,2, \cdots, n)
\end{gathered}
$$

For new customers in Market $\beta$, their WTP for NP is uniformly distributed on $\left[0, p_{N}\right]$ with population in Market $\beta$ is $M-D_{O N}$. As WTP and $p_{N}$ are unchanged, none of them will buy NP. While some of them will buy RPs instead, specifically, when $v_{N}>v_{\beta}^{\prime \prime}=\frac{p_{\beta R}}{\delta}$. Thus the demand for NP and RP in Market $\beta$ are

$$
\begin{gathered}
D_{\beta N}=0 \\
D_{\beta R}=\left(M-D_{O N}\right) \frac{p_{N}-v_{\beta}^{\prime \prime}}{p_{N}}=M\left(p_{N}-\frac{p_{\beta R}}{\delta}\right)
\end{gathered}
$$

The customer's options and the demands for NP and RP in both periods in segmented markets are displayed in Fig.2.

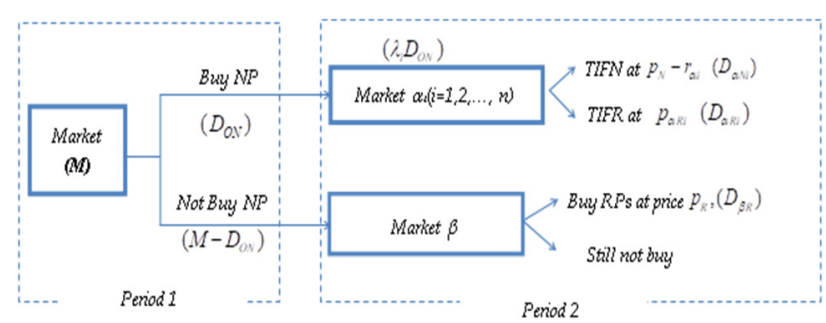

Figure 2. Customer's decisions and segmented market demands in both periods

\section{Model Analysis}

In a tiered remanufacturing system, UP with higher quality takes higher priority to be remanufactured because of less cost. While in the collection and sales market, the trade-in activities with lower quality UP returned or NP \& RP purchases without UP returned generate more cash flow. With consideration of the supply and demand of RP, to keep balance is very important in pricing strategy study.

An integrated optimization scheme is to price with the aim to maximize the total profit without restriction of collection, remanufacturing and sale among different tier. Tiered optimization scheme is to price with consideration 
of the RP demand and supply in each tier and set weights on each tier. Note that the replacement prices $p_{\alpha R i}(i=$ $1,2, \ldots, n)$ in Market $\alpha_{i}$ are not just the differences between the RP prices $P_{\beta R}$ and general return incentives $r_{\alpha i}$. In order to get the satisfactory collection, remanufacturing and sale strategy, we need to discuss the optimal $p_{N}, p_{\alpha R i}$ and $p_{\beta R}$ with subsidy proportion $\theta$ under two schemes. We first compute the equilibrium solution under integrated optimization scheme in Section 4.1, then under tiered optimization scheme by multi-objective programming in Section 4.2.

\subsection{Tiered pricing strategy under integrated optimization}

In tiered remanufacturing system, UP with higher quality takes higher priority to be remanufactured because of less cost. Under a scheme of integrated optimization, there is no restriction of RP supply among different tier, but that the total RP supply to Market $\alpha_{i} \& \beta$ is no more than the amount of remanufacturable UPs from Market $\alpha_{i}$.

To maximize the total profit, we solve the following model (6) with Eq.(1) to Eq.(5).

$$
\begin{gathered}
\max _{\left(p_{N}, p_{\alpha R l}, p_{\alpha R 2}, \ldots, p_{\alpha R N}, p_{\beta R}, k\right)} \pi^{I}=\left(p_{N}-c_{N}\right) D_{O N}+\left\{\sum_{i=1}^{n}\left[\left(p_{N}-r_{\alpha i}\right) D_{\alpha N i}+p_{\alpha R i} D_{\alpha R i}\right]+p_{\beta R} D_{\beta R}\right\} \\
-c_{N} \sum_{i=1}^{n} D_{\alpha N i}-\left[c_{\alpha k}\left(\sum_{i=1}^{n} D_{\alpha R i}+D_{\beta R}\right)-\sum_{i=1}^{k-1} \omega_{i}\left(c_{\alpha k}-c_{\alpha i}\right)\left(D_{\alpha N i}+D_{\alpha R i}\right)\right] \\
\text { S.T. } \sum_{i=1}^{k-1} \omega_{i}\left(D_{\alpha N i}+D_{\alpha R i}\right)<\sum_{i=1}^{n} D_{\alpha R i}+D_{\beta R} \leq \sum_{i=1}^{k} \omega_{i}\left(D_{\alpha N i}+D_{\alpha R i}\right) \\
;\left(k=1,2, \cdots, n k=1,2, \cdots, n^{1}\right) \\
\quad p_{N}>0, p_{\beta R}-r_{\alpha n} \geq p_{\alpha R n} \geq \cdots \geq p_{\alpha R 1} \geq \\
0 ; D_{\alpha N i}, D_{\alpha R i}, D_{\beta R} \geq 0(i=1,2, \cdots, n)
\end{gathered}
$$

Let $\sum_{i=1}^{k-1} \omega_{i}\left(c_{\alpha k}-c_{\alpha i}\right)\left(D_{\alpha N i}+D_{\alpha R i}\right)$ to be 0 , if $k=1$.

The first term $\left(p_{N}-c_{N}\right) D_{O N}$ represents the profit in Period 1; the second term $\sum_{i=1}^{n}\left[\left(p_{N}-r_{\alpha i}\right) D_{\alpha N i}+\right.$ $\left.p_{\alpha R i} D_{\alpha R i}\right]+p_{\beta R} D_{\beta R}$ stands for the revenue of Market $\alpha_{i} \& \beta$ in Period 2; $c_{N} \sum_{i=1}^{n} D_{\alpha N i}$ is the total manufacturing cost for NPs in Period 2; $c_{\alpha k}\left(\sum_{i=1}^{n} D_{\alpha R i}+D_{\beta R}\right)-$ $\sum_{i=1}^{k-1} \omega_{i}\left(c_{\alpha k}-c_{\alpha i}\right)\left(D_{\alpha N i}+D_{\alpha R i}\right) \quad$ denotes the remanufacturing cost if the lowest remanufactured quality level of UPs is $L_{k}$. The inequality constrain $\quad c_{\alpha k}\left(\sum_{i=1}^{n} D_{\alpha R i}+D_{\beta R}\right)-\sum_{i=1}^{k-1} \omega_{i}\left(c_{\alpha k}-\right.$ $\left.c_{\alpha i}\right)\left(D_{\alpha N i}+D_{\alpha R i}\right)$ specifies the lowest level UPs introduced in remanufacturing as the higher quality UPs are always preferred to be remanufactured. Besides, we set $p_{\beta R}-r_{\alpha i} \geq p_{\alpha R i} \geq 0$ for market order maintenance. In order to solve model (6), we take the following steps.

Step 1: Set up model (7-k) as followed, and let $k=1$.

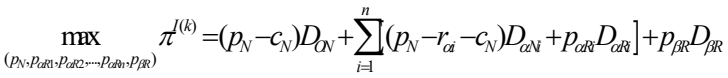

$$
\begin{aligned}
& -c_{\alpha k}\left(\sum_{i=1}^{n} D_{\alpha R k}+D_{\beta R}\right)+\sum_{i=1}^{k-1} \omega\left(c_{\alpha k}-c_{o i}\right)\left(D_{\alpha k i}+D_{\alpha k j}\right)
\end{aligned}
$$

S.T. $\quad p_{N}>0, p_{\beta R}-r_{\alpha n} \geq p_{\alpha R n} \geq \ldots \geq p_{\alpha R 1} \geq$ $0 ; D_{\alpha N i}, D_{\alpha R i}, D_{\beta R} \geq 0(i=1,2, \cdots, n)$

Step 2: Solve model $(7-k)$ and get optimal solution $\left(p_{N}^{I(k)}, p_{\alpha R 1}^{I(k)}, p_{\alpha R 2}^{I(k)}, \ldots, p_{\alpha R n}^{I(k)}, p_{\beta R}^{I(k)}\right)$, substitute it into Eq.(2) to Eq.(5), then we have $\left(D_{\alpha N i}^{I(k)}, D_{\alpha R i}^{I(k)}, D_{\beta R}^{I(k)}\right)$ for $i=1,2, \cdots, n$.

Step 3: Check if $\quad \sum_{i=1}^{n} D_{\alpha R i}^{I(k)}+D_{\beta R}^{I(k)}>$ $\sum_{i=1}^{k} \omega_{i}\left(D_{\alpha N i}^{I(k)}+D_{\alpha R i}^{I(k)}\right) \& k<n$, then let $k=k+1$ and turn back to Step 2 again. Else if $\sum_{i=1}^{n} D_{\alpha R i}^{I(k)}+D_{\beta R}^{I(k)} \leq$ $\sum_{i=1}^{k} \omega_{i}\left(D_{\alpha N i}^{I(k)}+D_{\alpha R i}^{I(k)}\right) \& k \leq n$, then we obtain the optimal solution already as $\left(p_{N}^{I *}, p_{\alpha R 1}^{I *}, p_{\alpha R 2}^{I *}, \ldots, p_{\alpha R n}^{I *}, p_{\beta R}^{I *}\right)=$ $\left(p_{N}^{I(k)}, p_{\alpha R 1}^{I(k)}, p_{\alpha R 2}^{I(k)}, \ldots, p_{\alpha R n}^{I(k)}, p_{\beta R}^{I(k)}\right)$. Else we need to solve model (8) and achieve $\left(p_{N}^{I *}, p_{\alpha R 1}^{I *}, p_{\alpha R 2}^{I *}, \ldots, p_{\alpha R n}^{I *}, p_{\beta R}^{I *}\right)=$ $\left(p_{N}^{I \prime}, p_{\alpha R 1}^{I \prime \prime}, p_{\alpha R 2}^{I \prime}, \ldots, p_{\alpha R n}^{I \prime}, p_{\beta R}^{I \prime}\right)$.

$$
\begin{aligned}
& \left.\max _{\left(p_{N}, p_{\alpha R R}, p_{\alpha R 2}, \ldots, p_{\alpha \beta R}, p_{\beta R R}\right)} \pi^{\prime^{\prime}}=\left(p_{N}-c_{N}\right) D_{O N}+\sum_{i=1}^{n}\left(p_{N}-r_{\alpha i}-c_{N}\right) D_{\alpha N i}+p_{\alpha R i} D_{\alpha R i}\right]+p_{\beta R} D_{\beta R} \\
& -c_{o n}\left(\sum_{i=1}^{n} D_{\alpha R i}+D_{\beta R}\right)+\sum_{i=1}^{n-1} \omega_{i}\left(c_{\alpha k}-c_{c i}\right)\left(D_{\alpha \lambda i}+D_{\alpha R i}\right)
\end{aligned}
$$

$$
\begin{aligned}
& \text { S.T. } \quad \sum_{i=1}^{n} D_{\alpha R i}+D_{\beta R}=\sum_{i=1}^{n} \omega_{i}\left(D_{\alpha N i}+D_{\alpha R i}\right) ; \\
& p_{N}>0, p_{\beta R}-r_{\alpha n} \geq p_{\alpha R n} \geq \ldots \geq p_{\alpha R 1} \geq \\
& 0 ; D_{\alpha N i}, D_{\alpha R i}, D_{\beta R} \geq 0(i=1,2, \cdots, n)
\end{aligned}
$$

By solving the model, we found that the marginal profit of selling RP in Market $\beta$ is more than that in Market $\alpha_{i}$, as the same order of price charged, owing to the amalgamated remanufacturing cost. Under integrated optimization scheme, there will be relatively higher $p_{\alpha R i}^{I *}$ and lower $p_{\beta R}^{I *}$ so that most of the old customers may opt to TIFN instead of TIFR and the customers in Market $\beta$ participate in a major way.

In order to maximize the total profit, tiered pricing strategy under integrated optimization depresses the old customer to accept RP despite of the assistance of government subsidy. Furthermore, it will discourage the old customer to return their old products in a long run and lead to the source reduction of remanufacturing, which causes unbalanced remanufacturing system.

${ }^{1}$ Let $\sum_{i=1}^{k-1} \omega_{i}\left(c_{\alpha k}-c_{\alpha i}\right)\left(D_{\alpha N i}+D_{\alpha R i}\right)$ to be 0 , if $k=1$. 


\subsection{Tiered pricing strategy under tiered optimization}

When seeking long-term profit and promoting the development of remanufacturing industry, a tiered optimization scheme is presented in this section. Thus, the profit optimization problem can be expressed as multiobjective programming with the same decision variables $\left(p_{N}, p_{\alpha R 1}, p_{\alpha R 2}, \ldots, p_{\alpha R n}, p_{\beta R}, k\right)$ as in $\operatorname{Model}(9)$ :

$$
\begin{gathered}
\left\{\begin{array}{c}
\max \pi_{j}^{T}=\left(p_{N}-r_{\alpha j}-c_{N}\right) D_{\alpha N j}+\left(p_{\alpha R j}-c_{\alpha j}\right) D_{\alpha R j} \quad(j=1,2, \ldots, n) \\
\max \pi_{n+1}^{T}=\left(p_{N}-c_{N}\right) D_{O N}+\left\{\sum_{i=1}^{n}\left[\left(p_{N}-r_{\alpha i}\right) D_{\alpha N i}+p_{\alpha R i} D_{\alpha R i}\right]+p_{\beta R} D_{\beta R}\right\} \\
-c_{N} \sum_{i=1}^{n} D_{\alpha N i}-\left[c_{\alpha k}\left(\sum_{i=1}^{n} D_{\alpha R i}+D_{\beta R}\right)-\sum_{i=1}^{k-1} \omega_{i}\left(c_{\alpha k}-c_{\alpha i}\right)\left(D_{\alpha N i}+D_{\alpha R i}\right)\right] \\
\operatorname{S.T} \sum_{i=1}^{j} D_{\alpha R i} \leq \sum_{i=1}^{j} \omega_{i}\left(D_{\alpha N i}+D_{\alpha R i}\right) ; \quad(j=1,2, \cdots, n)
\end{array}\right. \\
\sum_{i=1}^{k-1} \omega_{i}\left(D_{\alpha N i}+D_{\alpha R i}\right)<\sum_{i=1}^{n} D_{\alpha R i}+D_{\beta R} \leq \sum_{i=1}^{k} \omega_{i}\left(D_{\alpha N i}+D_{\alpha R i}\right) \quad(k=1,2, \cdots, n) \\
p_{N}>0, p_{\beta R}-r_{\alpha n} \geq p_{\alpha R n} \geq \ldots \geq p_{\alpha R 1} \geq 0 ; D_{\alpha N i}, D_{\alpha R i}, D_{\beta R} \geq 0(i=1,2, \cdots, n)
\end{gathered}
$$

We have $(n+1)$ objectives in model (9). The first objective function when $j=1$ denotes the total profit from Market $\alpha_{1}$ with the tiered remanufacturing system of highest quality UPs; the other objective function for any $j(j \leq n)$ signifies the profit from Market $\alpha_{j}$ with the support of corresponding tiered remanufacturing systems of related quality level UPs, and extra RPs made by higher level $L_{i}(i<j)$ could also be offered to Market $\alpha_{j}$ under the constraints of $\sum_{i=1}^{j} D_{\alpha R i} \leq \sum_{i=1}^{j} \omega_{i}\left(D_{\alpha N i}+D_{\alpha R i}\right)$. The $(n+1)^{\text {th }}$ objective and other constrains are equivalent to model (6) to acquire relatively higher total profit eventually. Thus, with given multi-objective weights, model (9) can be solved in sequence of $k$ as followed.

Step 1: Set up multi-object models (10-k) as followed, and let $k=1$.

$$
\begin{gathered}
\left\{\begin{array}{c}
\max \pi_{j}^{T(k)}=\left(p_{N}-r_{\alpha j}-c_{N}\right) D_{\alpha N j}+\left(p_{\alpha R j}-c_{\alpha j}\right) D_{\alpha R j} ;(j=1,2, \ldots, n) \\
\max \pi_{n+1}^{T(k)}=\left(p_{N}-c_{N}\right) D_{o N}+\left\{\begin{array}{c}
\sum_{i=1}^{n}\left[\left(p_{N}-r_{\alpha i}\right) D_{\alpha N i}+p_{\alpha R i} D_{\alpha R i}\right] \\
+p_{\beta R} D_{\beta R}
\end{array}\right\} \\
-c_{N} \sum_{i=1}^{n} D_{\alpha N i}-\left[\begin{array}{c}
c_{\alpha n}\left(\sum_{i=1}^{n} D_{\alpha R i}+D_{\beta R}\right)- \\
\sum_{i=1}^{n-1} \omega_{i}\left(c_{\alpha k}-c_{\alpha i}\right)\left(D_{\alpha N i}+D_{\alpha R i}\right)
\end{array}\right] \\
\text { S.T. } \quad \sum_{i=1}^{j} D_{\alpha R i} \leq \sum_{i=1}^{j} \omega_{i}\left(D_{\alpha N i}+D_{\alpha R i}\right) ;(j=1,2, \cdots, n) \\
\sum_{i=1}^{n} D_{\alpha R i}+D_{\beta R}=\sum_{i=1}^{n} \omega_{i}\left(D_{\alpha N i}+D_{\alpha R i}\right) ;
\end{array}\right. \\
p_{N}>0, p_{\beta R}-r_{\alpha n} \geq p_{\alpha R n} \geq \ldots \geq p_{\alpha R 1} \geq 0 ; D_{\alpha N i}, D_{\alpha R i}, D_{\beta R} \geq 0(i=1,2, \cdots, n)
\end{gathered}
$$

Step 2: Solve model $(10-k)$ with given multi-objective weights and get optimal solution $\left(p_{N}^{T(k)}, p_{\alpha R 1}^{T(k)}, p_{\alpha R 2}^{T(k)}, \ldots, p_{\alpha R n}^{T(k)}, p_{\beta R}^{T(k)}\right)$, substitute it into Eq.(2) to Eq.(5), then we have $\left(D_{\alpha N i}^{T(k)}, D_{\alpha R i}^{T(k)}, D_{\beta R}^{T(k)}\right)$ for $i=1,2, \cdots, n$.

Step 3: Check if $\sum_{i=1}^{n} D_{\alpha R i}^{T(k)}+D_{\beta R}^{T(k)}>$ $\sum_{i=1}^{k} \omega_{i}\left(D_{\alpha N i}^{T(k)}+D_{\alpha R i}^{T(k)}\right) \& k<n$, then let $k=k+1$ and turn back to Step 2 again. Else if $\sum_{i=1}^{n} D_{\alpha R i}^{T(k)}+D_{\beta R}^{T(k)} \leq$ $\sum_{i=1}^{k} \omega_{i}\left(D_{\alpha N i}^{T(k)}+D_{\alpha R i}^{T(k)}\right) \& k \leq n$, then we obtain the optimal solution already as $\left(p_{N}^{T *}, p_{\alpha R 1}^{T *}, p_{\alpha R 2}^{T *}, \ldots, p_{\alpha R n}^{T *}, p_{\beta R}^{T *}\right)=$ $\left(p_{N}^{T(k)}, p_{\alpha R 1}^{T(k)}, p_{\alpha R 2}^{T(k)}, \ldots, p_{\alpha R n}^{T(k)}, p_{\beta R}^{T(k)}\right)$. Else we need to solve model (11) and achieve $\left(p_{N}^{T *}, p_{\alpha R 1}^{T *}, p_{\alpha R 2}^{T *}, \ldots, p_{\alpha R n}^{T *}, p_{\beta R}^{T *}\right)=$ $\left(p_{N}^{T^{\prime}}, p_{\alpha R 1}^{T^{\prime}}, p_{\alpha R 2}^{T^{\prime}}, \ldots, p_{\alpha R n}^{T^{\prime}}, p_{\beta R}^{T^{\prime}}\right)$.

$$
\begin{gathered}
\left\{\begin{array}{c}
\max \pi_{j}^{T^{\prime}}=\left(p_{N}-r_{\alpha j}-c_{N}\right) D_{\alpha N j}+\left(p_{\alpha R j}-c_{\alpha j}\right) D_{\alpha R j} ;(j=1, \ldots, n) \\
\max \pi_{n+1}^{T^{\prime}}=\left(p_{N}-c_{N}\right) D_{o N}+\left\{\sum_{i=1}^{n}\left[\left(p_{N}-r_{\alpha i}\right) D_{\alpha N i}+p_{\alpha R i} D_{\alpha R i}\right]+p_{\beta R} D_{\beta R}\right\} \\
-c_{N} \sum_{i=1}^{n} D_{\alpha N i}-\left[c_{\alpha n}\left(\sum_{i=1}^{n} D_{\alpha R i}+D_{\beta R}\right)-\sum_{i=1}^{n-1} \omega_{i}\left(c_{\alpha k}-c_{\alpha i}\right)\left(D_{\alpha N i}+D_{\alpha R i}\right)\right] \\
\operatorname{S.T} \sum_{i=1}^{j} D_{\alpha R i} \leq \sum_{i=1}^{j} \omega_{i}\left(D_{\alpha N i}+D_{\alpha R i}\right) ;(j=1,2, \cdots, n)
\end{array}\right. \\
p_{N}>0, p_{\beta R}-r_{\alpha n} \geq p_{\alpha R n} \geq \ldots \geq p_{\alpha R 1} \geq 0 ; D_{\alpha N i}, D_{\alpha R i}, D_{\beta R} \geq 0(i=1,2, \cdots, n)
\end{gathered}
$$


The weight of multi-object $w_{n+1}=\left[a_{1}, a_{2}, \ldots, a_{n}, b\right]$ (balance weight) will reflect the RP promotion degree for Market $\alpha_{i}$ and $\beta$ so as to affect the effectiveness of TIFR subsidy. Specifically, model(9) is equivalent to model (6) with weight $w_{n+1}=[0,0, \ldots, 0,1]$ and only put value on integrated profit, which attracts new comers particularly and lower the impact of TIFR subsidy. While an even weight (eg. $\left.w_{n+1}=\operatorname{ones}(1, n+1)\right)$ addresses both concerns on tiered NP-RP equilibrium and total cross-tier profit where TIFR subsidy can play a role to amplify the old customer's surplus of TIFR.

Consider a special case of best circumstance with all remanufacturable ratio $\omega_{i}=1(i=1,2, \ldots n)$ and even weight $w_{n+1}=$ ones $(1, n+1)$, which implies all the UPs collected in Market $\alpha_{i}$ can be remanufactured, thus the RP supply for Market $\alpha_{i}$ is sufficient from its tier and all the extra RPs will flow to Market $\beta$. In this case, optimal pricing strategy $\left(p_{N}^{O T *}, p_{\alpha R 1}^{O T *}, p_{\alpha R 2}^{O T *}, \ldots, p_{\alpha R n}^{O T *}, p_{\beta R}^{O T *}\right)$ satisfy Eq.(12)\&(13). There are three terms in Eq.(13), the first is set to maintain the market order, the second is the optimal interior point, and the third ensures the supply to Market $\beta$ is no more than total extra RPs from all the Market $\alpha_{i}$.

$$
\begin{gathered}
(1-\theta+\delta) p_{N}^{O T *}-2(1-\theta) p_{\alpha R i}^{O T *}-(2-\theta) r_{\alpha i} \\
-(1-\theta)\left(c_{N}-c_{i}\right)=0 \quad(i \\
=1,2, \ldots, n) \\
p_{\beta R}^{O T *}=\max \left(p_{\alpha R n}^{O T *}, \frac{\delta p_{N}^{O T *}+c_{n}}{2}, \delta p_{N}^{O T *}\right. \\
\left.-\frac{\delta \sum_{i=1}^{n} D_{\alpha N i}^{O T *}}{M}\right)
\end{gathered}
$$
that:

The implicit differentiation based on Eq.(12) tells us

$$
\frac{d p_{\alpha R i}^{O T *}}{d \theta}=\frac{2 p_{\alpha R i}^{O T *}+r_{\alpha i}-c_{i}-\left(p_{N}^{O T *}-c_{N}\right)}{2(1-\theta)}>0 \quad(i=
$$$$
1,2, \ldots, n)^{2}
$$

$$
\frac{d p_{N}^{O T *}}{d \theta}=-\frac{2 p_{\alpha R i}^{O T *}+r_{\alpha i}-c_{i}-\left(p_{N}^{O T *}-c_{N}\right)}{1-\theta+\delta}<0 \quad(i=
$$

According to Eq.(14) \& Eq.(15), with the increase of subsidy ratio, the replacement price for each Market $\alpha_{i}$ goes up a little bit because the subsidy removes parts of the price pressure of TIFR customers, and the sales price for NP drops and attracts more users in Period 1 so that scales of Market $\alpha_{i}$ in Period 2 expand. And if the RPs are sufficient enough for the demand in Market $\beta$ to make sure that $p_{\beta R}^{O T *}=\frac{\delta p_{N}^{O T *}+c_{n}}{2}$, then the RP's sales price will go down to draw more new buyers. Otherwise, $p_{\beta R}^{O T *}$ will rise with RP supply constraints. Generally, since the new customer gets no subsidy while the old customer does by TIFR, the demand for RPs will shrink in Market $\beta$ and grow in Market $\alpha_{i}$ with limited RP supply in Period2

\section{NUMERICAL ANALYSIS}

Based on the data survey available by Volkswagen and Caterpillar, we set 3 level of UPs as and basic Market scale . Since higher quality UP holder has relatively lower enthusiasm to trade-in, the proportions of population belonging to Market are set to be . The Remanufacturable proportions of 3 levels are to get RPs with quality and performance generally comparable to that of NPs. Considering is distributed on $[0,1]$, we set cost of a NP is 0.6 . In that total cost of remanufacturing, including return incentive and unit RP cost, is usually 50\% lower than that of NP, RP costs are and corresponding collection incentives are in this section. As the TIFR policy mainly aims for functional products, e.g. auto-part and mechanical equipment, we initially set $\delta=0.5$ according to the related investigation (Sojump, n.d.) and analysis and $\theta=0.1$ as stated in related documents [32].

We first conduct pricing optimization scheme comparison on subsidy effect in Section 5.1, then compare the impacts of other parameter changes on model performance, specifically, between customer's receptiveness to RP and subsidy ratio in Section 5.2.

\subsection{Subsidy effect based on Integrated and Tiered optimization Scheme}

Given the basic model and parameters setting above, we obtain the optimal strategies with and without TIFR subsidy under integrated \& tiered optimization schemes, as shown in Table 1 .

Table 1. The subsidy effect comparison between Integrated and Tiered optimization Scheme.

\begin{tabular}{|l|l|l|l|l|}
\hline \multirow{2}{*}{$\begin{array}{l}\text { Optimization } \\
\text { Scheme }\end{array}$} & \multicolumn{2}{|l|}{ Integrated } & \multicolumn{2}{l|}{ Tiered } \\
\cline { 2 - 5 } & $\theta=0$ & $\theta=0.1$ & $\theta=0$ & $\theta=0.1$ \\
\hline$p_{N}$ & 0.799 & 0.793 & 0.799 & 0.790 \\
\hline$p_{\alpha R 1}$ & 0.280 & 0.307 & 0.205 & 0.213 \\
\hline$p_{\alpha R 2}$ & 0.320 & 0.336 & 0.280 & 0.290 \\
\hline$p_{\alpha R 3}$ & 0.331 & 0.336 & 0.343 & 0.347 \\
\hline$p_{\beta R}$ & 0.371 & 0.376 & 0.383 & 0.387 \\
\hline$D_{O N}$ & 100.067 & 103.704 & 100.001 & 105.007 \\
\hline$D_{\alpha N 1}$ & 10.007 & 10.370 & 2.500 & 2.150 \\
\hline$D_{\alpha N 2}$ & 20.014 & 17.941 & 12.000 & 8.350 \\
\hline$D_{\alpha N 3}$ & 21.245 & 14.912 & 24.808 & 10.201 \\
\hline$D_{\alpha R 1}$ & 0 & 0 & 7.500 & 10.801 \\
\hline$D_{\alpha R 2}$ & 2.780 & 0 & 8.000 & 18.705 \\
\hline$D_{\alpha R 3}$ & 8.775 & 16.199 & 5.192 & 12.797 \\
\hline$D_{\beta R}$ & 29.251 & 20.409 & 17.308 & 7.954 \\
\hline
\end{tabular}

Influenced by TIFR subsidy, the replacement prices for $\mathrm{RP}$ in all segments are raised in Period 2, but the prices under Tiered scheme increase less. The sales price for NP

\footnotetext{
${ }^{2}$ Generally speaking, the remanufacturing has higher margin than traditional manufacturing, that is, $p_{\alpha R i}+r_{\alpha i}-c_{i}>p_{N}-c_{N}$, which is the basic condition for a remanufacturing industry.
} 
drops a little to attract more buys in Period 1 so as to expand the scale of recycling markets in Period 2. The TIFR demands from old customers are enlarged by providing subsidy, while the raising proportions are different under integrated or tiered optimization schemes.

Based on the remanufacturing priority of quality levels, the total cost of remanufacturing is relatively stable no matter where the RPs flow to. Integrated pricing scheme values much on unit margin of RP, so it prefers attracting new comers and neglects the needs of old customers. For example, the TIFR price for old customers in Market $\alpha_{1}$ is 0.280 unit $\left(p_{\alpha R 1}^{I *}(\theta=0)\right)$ if returning a good quality UP worth at least $0.120 \operatorname{unit}\left(r_{\alpha 1}\right)$, while the RP price without return is 0.387 unit $\left(p_{\beta R}^{I *}(\theta=0)\right)$ in Market $\beta$, which kill the TIFR motivation of these old customers. Under this circumstance, TIFR subsidy can do little to mobilize the old customer's enthusiasm, particularly for higher quality UP holders. Specifically, with given $p_{\alpha R 1}^{I *}(\theta=0.1)=$ 0.307 , the out-of-pocket cost is $(1-\theta) p_{\alpha R 1}^{I *}(\theta=0.1)=$ 0.276 for those choosing TIFR in Market $\alpha_{1}$. Considering the UP returned (0.120), the total cost for them is still more than $p_{\beta R}^{T *}(\theta=0.1)(0.376)$.

Actually, it is expected that $P_{\alpha R i}$ should be no more than $P_{\beta R}-r_{\alpha i}$ so as to encourage old customers to trade in their UPs. When pricing under tiered optimization scheme, the replacement prices in Market $\alpha_{i}$ of higher quality levels are cut a lot, e.g. $p_{\alpha R 1}^{T *}(\theta=0)$ is 0.205 unit, compared to $p_{\beta R}^{T *}(\theta=0)=0.383\left(>r_{\alpha 1}+p_{\alpha R 1}^{T *}(\theta=0)\right)$, which is inspiring and boosts the TIFR demand in those segments. Since only customers in Market $\alpha_{i}$ are offered TIFR subsidy, tiered optimization scheme plays a great role in balancing the remanufacturing system. For instance, the out-of-pocket cost is $(1-\theta) p_{\alpha R 1}^{T *}(\theta=0.1)=0.192$ for those choosing TIFR in Market $\alpha_{1}$, thus the total cost for them $(0.192+0.120=0.312)$ is far less than $p_{\beta R}^{T *}(\theta=$ $0.1)(0.387)$. Therefore, tiered optimization scheme is necessary to arouse old customer's enthusiasm, form a benign environment for TIFR and achieve stability of remanufacturing development in the long run.

\subsection{Subsidy effect combined with Receptiveness to RP}

In this section, we want to study the impact of subsidy ratio $(\theta)$ combined with the changing exogenous variable $\delta$ on RP demands in different market segments. Based on the analysis in Section 5.1, we discuss the optimal strategies under tiered optimization scheme.

As the ratio of consumers' WTP between RP and NP, $\delta$ reflects the customer's receptiveness to RP. It will rise with the advance of remanufacturing technology and consumer environmental awareness. Generally speaking, there will be more customers switching to RP purchase with the increase of $\delta$. Under the environment of limited UP returns, the effects of improving customer's RP receptiveness are shown in Fig.3(a) \&(b).

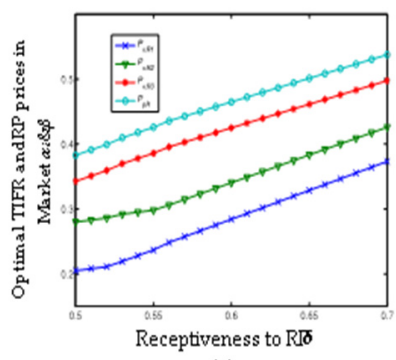

(a)

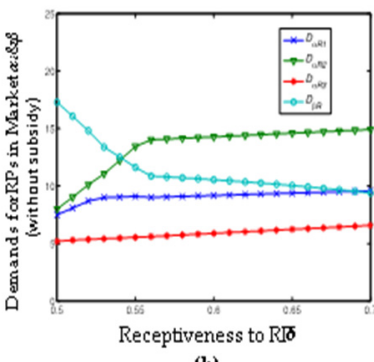

(b)
Figure 3. Optimal pricing strategy and corresponding Demands for RPs with varying receptiveness to RP $(\boldsymbol{\delta})$ in Market $\alpha_{i} \& \beta$ (without subsidy $\theta=0$ )

When $\delta$ increases, all the optimal TIFR prices and RP sales price rise (Fig.3(a)) without reducing the corresponding TIFR demand $\left(D_{\alpha R 1}, D_{\alpha R 2} \& D_{\alpha R 3}\right.$ keep climbing in Fig.3(b)) while the demand for RP in Market $\beta$ drops $\left(D_{\beta R}\right.$ keeps falling in Fig.3(b)) because of the limited returns of UPs under tiered optimization scheme. Although the consequence is ideal, customer's receptiveness to RP cannot be improved overnight. An alternative measure to accelerate the development of remanufacturing industry is establishing subsidy mechanism.

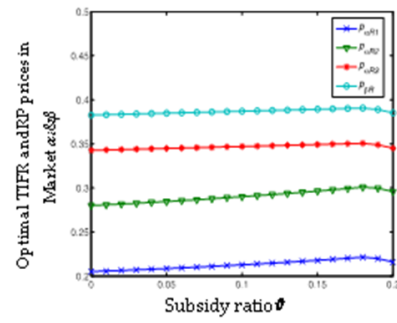

(a)

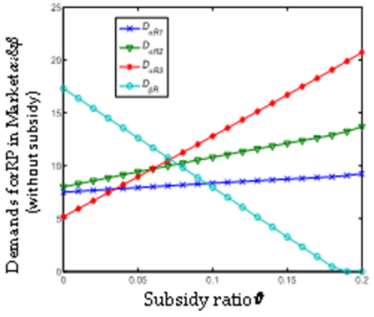

(b)
Figure 4. Optimal pricing strategy and corresponding Demands for RP with varying subsidy $(\theta)$ in Market $\alpha_{i} \& \beta(\delta=0.5)$

As shown in Fig. 4(a), the optimal TIFR and RP prices grow slowly with increasing $\theta$, however, the demands for RP in Market $\alpha_{i}$ upsurge dramatically. Specifically, TIFR subsidy cuts more replacement price for old customers who return low quality UPs because the original replacement price is higher. Thus it stimulates more TIFR demand in those segments $\left(D_{\alpha R 3}\right.$ grows fastest in Fig. 4(b)). When $\theta$ exceeds the threshold, the TIFR demands will cover all the RP supply, and there is no need to price $\mathrm{RP}$ in Market $\beta$ by then.

Considering the consistent of a policy that the subsidy ratio can't change very often, we then study the pricing and corresponding demand of varying $\delta$ with fixed $\theta=$ $10 \%$. We set the start $\delta$ as 0.4 , and find the demands of TIFR ascend faster in Fig. 5(b) than in Fig.3(b) with close price changes (Fig. 5(a) and Fig.3(a)). When we take efforts to improve the customer's receptiveness to RP, the $\mathrm{RP}$ market structure is improved more effective with the help of TIFR subsidy. 


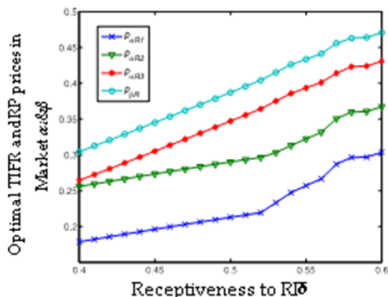

(a)

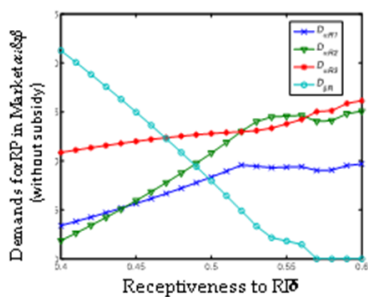

(b)
Figure 5. Optimal pricing strategy and corresponding Demands for RP with varying receptiveness to RP $(\delta)$ in Market $\alpha_{i} \& \beta$ ( subsidy rate $\theta=0.1$ )

In conclusion, integrated optimization scheme with tiered pricing neglects the experience of old customers and is not conducive to the development of remanufacturing. Tiered optimization scheme sets objectives for each tier and safeguards the interests of each tier respectively. Not only the future product returns, but also brand effectiveness can be enhanced by that. Both the increases of receptiveness to RP $(\boldsymbol{\delta})$ and subsidy ratio $(\boldsymbol{\theta})$ boost TIFR demand of UP holders with tiered optimization. And with the help of subsidy, the input to improve customers' receptiveness to RP will have effects more pronounced.

\section{Conclusions}

Multiple refund policy with a small number of quality classes already brings major advantages, but tiered TIFR pricing still needs more discussion, not only to maximize the profit but also to balance the UP collection and RP demand.

We study the quality-based pricing problem with government subsidy under integrated and tiered optimization scheme. After examining the tiered quality collection \& remanufacturing system and analyzing market demand, we develop profit models and use multi-object programming to determine the balanced optimal prices and production quantities.

Since TIFR subsidy is a relatively new business model, our research fills the gaps between the practice and the literature in this domain and draws the following managerial implications:

1) It is beneficial to employ the tiered remanufacturing approach and to offer tiered pricing strategy, especially when remanufacturing cost differs considerably due to different quality of UPs. Generally, higher quality UP will retain more key functions and incur less remanufacturing cost, thus the company will remanufacture under a quality priority sequence no matter where the RPs flow to.

2) Since the integrated optimization scheme emphasizes the total profit across all the tiers, it prefers inviting low WTP new comers for RP because of higher profit margin with relative stable total remanufacturing cost. And it drives old customers to TIFN instead with a comparative higher TIFR price. This is not helpful for balancing remanufacturing system and the company's brand image consolidation in the long run. Tiered optimization scheme with multiple objectives is therefore a satisfactory alternative under this circumstance.

3) Subsidy works well accompanied by tiered optimization scheme. It enhances the result for attracting more UPs and promoting RP demand by reducing the TIFR expenditure of old customers. The rise of customer's receptiveness to RP will enlarge the demand in TIFR market, but market structure can only be upgraded in a certain degree. With the help of subsidy, the rise of customer's receptiveness can gain more achievements in market structure improvement.

For future research, we will study remanufacturing system balance with competition between the manufacturer and the remanufacturer by considering quality disparity and TIFR subsidy. We will also consider coordination issue under supply chain setting with retailers responsible for selling and offering trade-in.

\section{ACKNOWLEDGMENT}

The authors would like to thank the editor and all anonymous referees for their valuable comments and suggestions. This work is supported by Innovation and Entrepreneurship Training Program of Reading Academy under Grant No. 1321182001001.

\section{ABBREVIATIONS}

The following abbreviations are used in this manuscript:

NP New Product

RP Remanufactured Product

UP Used Product

PLC Product life cycle

TIFN Trade In For NP

TIFR Trade In For RP

WTP Willing to Pay

NDRC National Development and Reform Commission

\section{REFERENCES}

1. "NDRC: Encourage recycling of used motor vehicle parts for remanufacturing," p. 2020, 2020.

2. “Brief News," Geostand. Newsl., vol. 19, no. 1, pp. 97-98, 1995, doi: 10.1111/j.1751908X.1995.tb00154.x.

3. "Analysis on the market status and Development Prospect of China's remanufacturing industry in 2020."

4. X. Yuan et al., "Resources, Conservation \& Recycling Transitioning China to a circular economy through remanufacturing: A comprehensive review of the management institutions and policy system," Resour. Conserv. Recycl., vol. 161, no. November 2019, p. 104920, 2020, doi: 10.1016/j.resconrec.2020.104920.

5. P. Ma, Y. Gong, and P. Mirchandani, "Trade-in for remanufactured products: Pricing with double reference effects," Int. J. Prod. Econ., vol. 230, no. June 2019, 2020, doi: 10.1016/j.ijpe.2020.107800.

6. X. Zhang, M. Zhang, H. Zhang, Z. Jiang, C. Liu, and W. Cai, "A review on energy, environment and economic assessment in remanufacturing based on life cycle assessment method," J. Clean. Prod., vol. 255, p. 120160, 2020, doi: 10.1016/j.jclepro.2020.120160. 
7. H. Qiao and Q. Su, "Impact of government subsidy on the remanufacturing industry," Waste Manag., no. xxxx, 2020, doi: 10.1016/j.wasman.2020.10.005.

8. Y. Zhou, Y. Xiong, and M. Jin, "Less is more: Consumer education in a closed-loop supply chain with remanufacturing," Omega (United Kingdom), no. xxxx, p. 102259, 2020, doi: 10.1016/j.omega.2020.102259.

9. Y. Xiong, G. Li, Y. Zhou, K. Fernandes, and R. Harrison, "Int . J . Production Economics Dynamic pricing models for used products in remanufacturing with lost-sales and uncertain quality," Intern. J. Prod. Econ., vol. 147, pp. 678-688, 2014, doi: 10.1016/j.ijpe.2013.04.025.

10. S. Wei, O. Tang, and W. Liu, "Int . J . Production Economics Refund policies for cores with quality variation in OEM remanufacturing," Intern. J. Prod. Econ., vol. 170, pp. 629-640, 2015, doi: 10.1016/j.ijpe.2014.12.006.

11. K. Li, J. Liu, H. Fu, and B. Liu, "Acquisition and pricing strategies in hybrid manufacturingremanufacturing systems," J. Manuf. Syst., vol. 57, no. October, pp. 217-230, 2020, doi: 10.1016/j.jmsy.2020.09.006.

12. Z. Liu, J. Chen, and C. Diallo, "Optimal production and pricing strategies for a remanufacturing firm," Intern. J. Prod. Econ., vol. 204, no. January, pp. 290315, 2018, doi: 10.1016/j.ijpe.2018.07.015.

13. A. D. V. Bittar, "Selling remanufactured products : Does consumer environmental consciousness matter?," J. Clean. Prod., vol. 181, pp. 527-536, 2018, doi: 10.1016/j.jclepro.2018.01.255.

14. Z. Liu, C. Diallo, J. Chen, and M. Zhang, "International Journal of Production Economics Optimal pricing and production strategies for new and remanufactured products under a non-renewing free replacement warranty," Int. J. Prod. Econ., vol. 226, no. July 2019, p. 107602, 2020, doi: 10.1016/j.ijpe.2019.107602.

15. R. Kleber, M. Reimann, G. C. Souza, and W. Zhang, "On the robustness of the consumer homogeneity assumption with respect to the discount factor for remanufactured products," Eur. J. Oper. Res., vol. 269, no. 3, pp. 1027-1040, 2018, doi: 10.1016/j.ejor.2018.02.052.

16. S. Gan, I. N. Pujawan, and B. Widodo, "Int . J . Production Economics Pricing decision for new and remanufactured product in a closed-loop supply chain with separate sales-channel," Intern. J. Prod. Econ., vol. 190, pp. 120-132, 2017, doi: 10.1016/j.ijpe.2016.08.016.

17. X. Han, Q. Yang, J. Shang, and X. Pu, "Optimal strategies for trade-old-for-remanufactured programs: Receptivity, durability, and subsidy," Int. J. Prod. Econ., vol. 193, no. January, pp. 602-616, 2017, doi: 10.1016/j.ijpe.2017.07.025.

18. L. Xiao, X. Wang, and K. Chin, "Trade-in strategies in retail channel and dual-channel closed-loop supply chain with remanufacturing," Transp. Res. Part E, vol.
136, no. March, p. 101898, 2020, doi: 10.1016/j.tre.2020.101898.

19. Y. Wang, X. Chang, Z. Chen, Y. Zhong, and T. Fan, "Impact of subsidy policies on recycling and remanufacturing using system dynamics methodology : a case of auto parts in China," J. Clean. Prod., vol. 74, pp. 161-171, 2014, doi: 10.1016/j.jclepro.2014.03.023.

20. J. Zhang, B. Yang, and M. Chen, "Challenges of the development for automotive parts remanufacturing in China," J. Clean. Prod., vol. 140, no. 800, pp. 10871094, 2017, doi: 10.1016/j.jclepro.2016.10.061.

21. T. Shu, Y. Wang, S. Chen, S. Wang, and K. K. Lai, "Decisions on Remanufacturing with WTP Disparity and Recycling Competition under Government Subsidies," 2017, doi: 10.3390/su9091503.

22. J. Cao, X. Chen, X. Zhang, Y. Gao, and X. Zhang, "Overview of remanufacturing industry in China: Government policies , enterprise , and public awareness," J. Clean. Prod., vol. 242, p. 118450, 2020, doi: 10.1016/j.jclepro.2019.118450.

23. X. Zhang, Q. Li, Z. Liu, and C. Chang, "Computers \& Industrial Engineering Optimal pricing and remanufacturing mode in a closed-loop supply chain of WEEE under government fund policy," Comput. Ind. Eng., no. September, p. 106951, 2020, doi: 10.1016/j.cie.2020.106951.

24. B. Liu et al., "The effect of remanufacturing and direct reuse on resource productivity of China's automotive production," J. Clean. Prod., vol. 194, pp. 309-317, 2018, doi: 10.1016/j.jclepro.2018.05.119.

25. Z. Wang, B. Li, X. Zhu, B. Xin, and Y. Wang, "The impact of donation subsidy of remanufactured products on Manufacturer 's pricing-production decisions and performances," J. Clean. Prod., vol. 202 pp. 892-903, 2018, doi: 10.1016/j.jclepro.2018.08.223.

26. Z. Liu, J. Tang, B. Li, and Z. Wang, "Trade-off between remanufacturing and recycling of WEEE and the environmental implication under the Chinese Fund Policy," J. Clean. Prod., vol. 167, pp. 97-109, 2017, doi: 10.1016/j.jclepro.2017.08.137.

27. Y. Chen, B. Li, G. Zhang, and Q. Bai, "Quantity and collection decisions of the remanufacturing enterprise under both the take-back and carbon emission capacity regulations," Transp. Res. Part E, vol. 141, no. December 2019, p. 102032, 2020, doi: 10.1016/j.tre.2020.102032.

28. J. Ding, W. Chen, and W. Wang, "Computers \& Industrial Engineering Production and carbon emission reduction decisions for remanufacturing firms under carbon tax and take-back legislation," Comput. Ind. Eng., vol. 143, no. November 2019, p. 106419, 2020, doi: 10.1016/j.cie.2020.106419.

29. T. Shu, Z. Peng, S. Chen, S. Wang, and K. K. Lai, "Government Subsidy for Remanufacturing or Carbon Tax Rebate: Which Is Better for Firms and a LowCarbon Economy," doi: 10.3390/su9010156. 
30. L. Wang and M. Chen, "Policies and perspective on end-of-life vehicles in China," J. Clean. Prod., vol. 44, no. 2013, pp. 168-176, 2015, doi: 10.1016/j.jclepro.2012.11.036.

31. W. Ma, Z. Zhao, and H. Ke, "Dual-channel closedloop supply chain with government consumptionsubsidy," Eur. J. Oper. Res., vol. 226, no. 2, pp. 221227, 2013, doi: 10.1016/j.ejor.2012.10.033.
32. C. Utilization, "The TIFR pilot scheme of remanufacturing products," pp. 5-7.

33. Sojump.. Questionnaire on perceived value of automotive engine remanufacturing products. http://www.sojump.com/viewstat/2269237.aspx, (11 2014) 